\title{
Identification of Fragmentation Channels of Dinucleotides Using Deuterium Labeling
}

\author{
Dorothée Balbeur, ${ }^{\mathrm{a}}$ Dominique Dehareng, ${ }^{\mathrm{b}}$ and Edwin De Pauw ${ }^{\mathrm{a}}$ \\ ${ }^{a}$ Laboratory of Mass Spectrometry, University of Liège, Liège, Belgium \\ ${ }^{\mathrm{b}}$ Center for Protein Engineering, University of Liège, Liège, Belgium
}

\begin{abstract}
The fragmentation of the totally deuterated dinucleotide $\mathrm{dAT}^{-}$in labile positions (heteroatombound hydrogens) was compared for different MS/MS methods: CID, IRMPD, and EID. These experiments allowed us to affirm the coexistence of several fragmentation channels. They can be classified according to the involvement of nonlabile or labile protons in the fragmentation process. Moreover, double resonance experiments were performed in IRMPD and EID. They demonstrated the existence of consecutive fragmentation processes. The probability with which each channel is taken depends on the fragmentation technique used, i.e., the energy and the time scale of the method. The fragmentation channels that involve labile protons requiring peculiar three-dimensional structures are entropically unfavorable and enthalpically favorable. They are more observed in IRMPD and EID. The involvement of labile and, therefore, exchangeable protons in the fragmentation mechanism casts doubt on the use of tandem mass spectrometry to localize incorporated deuteriums in oligonucleotides. (J Am Soc Mass Spectrom 2010, 21, 23-33) (c) 2010 Published by Elsevier Inc. on behalf of American Society for Mass Spectrometry
\end{abstract}

$\mathrm{O}$ ligonucleotide ions can be formed using different ionization methods, the most frequently used being those of electrospray ionization (ESI) and matrix assisted laser desorption ionization (MALDI). A wide range of activation methods can also be applied to nucleic acid ions for MS/MS experiments, including collision induced dissociation (CID), infrared multiphoton dissociation (IRMPD), blackbody infrared radiative dissociation (BIRD), sustained off-resonance irradiation (SORI), and more recently, fragmentation methods involving electron-ion interactions, such as electron capture dissociation (ECD), electron detachment dissociation (EDD), and electron induced dissociation (EID). The knowledge of detailed dissociation channels of oligonucleotides is essential for successful fragmentation pattern recognition and fragment ion prediction. This can also help to assess the use of tandem mass spectrometry to localize the incorporated deuteriums following hydrogen/deuterium exchange experiments. The deuterium migration during ion activation and fragmentation has been actively discussed for peptides [1-5], but not for oligonucleotides. To contribute to the understanding of the fragmentation mechanism, we examined, in this paper, the fragmentation of a totally deuterated dinucleotide, $\mathrm{dAT}^{-}$, in labile positions (heteroatom-bound hydrogens). The main aim of the work was to determine which hydrogens are involved in the fragmentation process.

Address reprint requests to Dorothée Balbeur, Laboratory of Mass Spectrometry, University of Liège, 3 Allée de la Chimie Bat. B6c, B-4000 Liège, Belgium. E-mail: dbalbeur@ulg.ac.be
Several fragmentation mechanisms were proposed to explain the formation of base loss, $\mathrm{w}$ and [a - B] ions (see McLuckey's nomenclature [6] in collision-activated dissociation [6]. These mechanisms, which are useful for later discussion, are illustrated in Figure 1, and are briefly described in this paragraph. In 1986, Cerny et al. [7] proposed a mechanism for loss of $\mathrm{B}^{-}$in fast atom bombardment (FAB) mass spectrometry, which was later supported by Phillips and McCloskey [8]. In this mechanism (Figure 1.1.1.), $\mathrm{B}^{-}$is lost by backside nucleophilic attack on $\mathrm{C}^{\prime}$ by negatively charged phosphate. Cerny et al. also suggested a mechanism for $\mathrm{w}$ ion formation (Figure 1.1.2.). Its process is induced by nucleophilic attack of the phosphate oxygen on the $2^{\prime}$-hydrogen via a six-membered ring intermediate. In 1993, McLuckey and Habibi-Goudarzi [9] suggested in ESI two 1,2-elimination processes to explain losses of neutral and negatively charged bases, and the subsequent formation of $[\mathrm{a}-\mathrm{B}]$ and $\mathrm{w}$ ions (Figure 1.2.). This was followed by Rodgers and coworkers [10], who proposed, in FAB, a proton-bound intermediate as the common precursor for losses of both neutral and charged bases (Figure 1.3.). In 1995, Barry et al. [11] proposed, in ESI, a two-step 1,2-elimination mechanism (Figure 1.4.) where the N-glycosyl bond cleavage is catalyzed by the phosphate group. In the same year, Nordhoff and coworkers [12] proposed a mechanism to explain fragmentation of MALDI-generated ions. This corresponds to a charge-remote, base-elimination mechanism catalyzed by intramolecular proton transfer followed by subsequent cleavage of the $3^{\prime}$ - and $5^{\prime}$ phosphoester bonds (Figure 1.5.1.). Nordhoff et al. also 


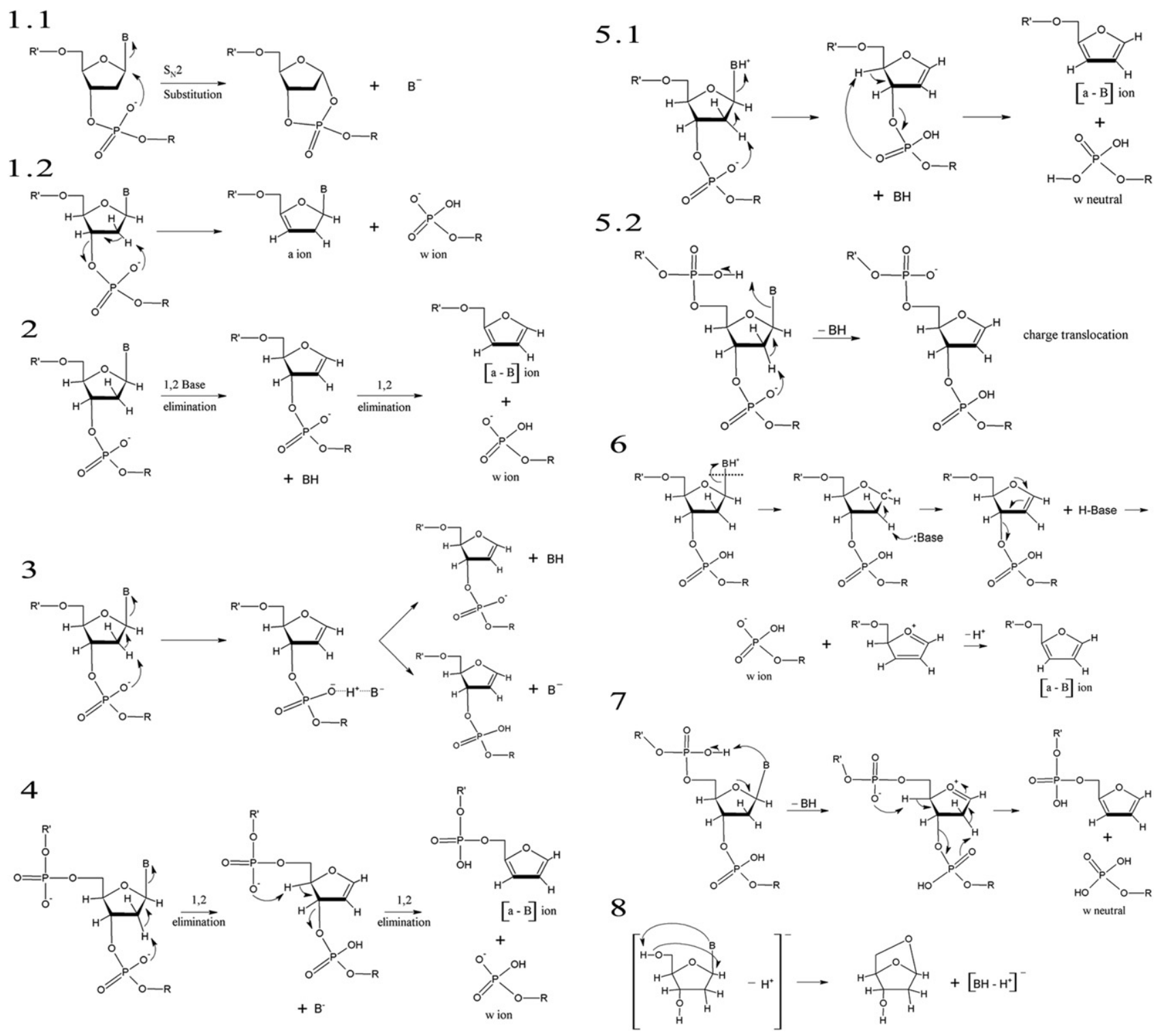

Figure 1. Summary of previously proposed fragmentation mechanisms.

explained the formation of a base-loss fragment thanks to a charge translocation mechanism (Figure 1.5.2.). Still, in MALDI, Zhu et al. [13] opted for proton transfer from the matrix to a nucleobase instead of intramolecular proton transfer as the initial step for base loss (Figure 1.6.). Wan and Gross [14-17] proposed other hydrogen atoms than the ones located on the deoxyribose ring for the proton transfer to the nucleobase. Thanks to H/D exchange $[15,16]$ and methylphosphonate substitution experiments [17] in ESI, Wan and Gross suggested a fragmentation mechanism involving proton transfer from the adjoining $5^{\prime}$-phosphate to the nucleobase. The zwitterionic $[\mathrm{M}-\mathrm{B}]^{-}$intermediate, which has a third charge site, serves as the common precursor for the formation of $[\mathrm{a}-\mathrm{B}]$ and the complementary w ions (Figure 1.7.). More recently, Liu et al. [18] proposed a novel mechanism of $\mathrm{N}$-glycosidic bond cleavage in nucleoside. Instead of a new double-bond generating between $\mathrm{C}^{\prime}$ ' and $\mathrm{C}^{\prime}$ ' as often previously reported, they suggested that with $H / D$ exchange experiments the proton of 5'-hydroxyl group on ribose moiety should be removed by the departing anionic nucleobase, resulting in a novel five-member ring via bond formation between the $5^{\prime}$-oxygen and $1^{\prime}$-carbon (Figure 1.8.).

The different mechanisms presented above can be classified in two categories: (1) the ones involving protons that are nonlabile and are bound to the carbon atoms of the ribose ring, and (2) those involving labile protons which are bound to phosphate and 5'-hydroxyl group on ribose moiety. These fragmentation mechanisms involving the transfer of labile protons are supposed to require particular three-dimensional conformations with spatial closeness between labile proton 
donor and acceptor sites to occur. In this work, the fragmentation of the totally deuterated dinucleotide $\mathrm{dAT}^{-}$in labile positions was compared for different MS/MS methods and discussed regarding the existence of the two categories. The different fragmentation techniques used were CID, IRMPD, and EID. $\mathrm{dAT}^{-}$was chosen following our previous study of gas-phase H/D exchange of dinucleotide ions [19]. Because it is a heterogenous dinucleotide [the $\mathrm{w}$ and $\mathrm{d}$ fragments, and (M-Base) ions have different masses] and because it is known to show a low number of different conformations, $\mathrm{dAT}^{-}$corresponds to a situation for which the fragmentation channels proposed to explain the data can be straightforwardly linked to conformations.

IRMPD is often described as an alternative vibrational excitation method [20, 21], providing similar oligonucleotides fragmentation patterns as CID. Because IRMPD is also a "slow heating" fragmentation technique, it is usually proposed to proceed through the same mechanism as in CID. Thanks to double resonance (DR) IRMPD experiments, Kinet et al. [22] and Yang et al. [23] supported the mechanism proposed by Gross and coworkers involving neutral base loss as the first step of the fragmentation process.

In the EDD experiment, irradiation of the precursor anion with $>10 \mathrm{eV}$ electrons is proposed to produce an excited intermediate that can undergo electron detachment (EDD) or direct decomposition (EID). EID originally referred to electron irradiation of singly charged anions, and has not been much applied to oligonucleotides [22]. However, its application to small molecules and peptides [24-26] has shown fragmentation similar to that of CID or IRMPD. Thanks to DR-EDD experiments, Kinet et al. [22] showed that, contrary to IRMPD, neutral base loss is not the first step of the fragmentation process.

For the purpose of obtaining more information about the fragmentation channels, especially the possible consecutive fragmentation channels, DR-IRMPD and DREID experiments were performed.

\section{Experimental}

\section{Sample Preparation}

The DNA dinucleotide, dAT, was obtained from Eurogentec (Seraing, Belgium). $\mathrm{D}_{2} \mathrm{O}$ and $\mathrm{CD}_{3} \mathrm{OD}$ were purchased from Euriso-Top (Gif sur Yvette, France). All these compounds were used without further purification. Totally deuterated dAT in labile positions was obtained by dissolving in $\mathrm{D}_{2} \mathrm{O}$. The injection solution was prepared by adding the appropriate amount of $\mathrm{CD}_{3} \mathrm{OD}$ to obtain a $\mathrm{CD}_{3} \mathrm{OD} / \mathrm{D}_{2} \mathrm{O}$ ratio of $15 / 85$ ( $\mathrm{vol} /$ vol) and a dinucleotide concentration of $10^{-5} \mathrm{M}$. The protonated dAT solution was obtained by following the same procedure with protonated solvents.

\section{Collision Induced Dissociation Experiments}

CID experiments were performed on a Q-TOF Ultima Global (Micromass UK Ltd., Manchester, UK) electrospray mass spectrometer. The z-spray was operated in the negative ion mode at a capillary voltage of $2.5 \mathrm{kV}$ and the solutions were infused at a flow rate of 240 $\mu \mathrm{L} / \mathrm{h}$. The source block temperature was $80^{\circ} \mathrm{C}$ and the desolvation gas $\left(\mathrm{N}_{2}\right)$ temperature was $120^{\circ} \mathrm{C}$. The $\mathrm{RF}$ Lens 1 voltage was set to $50 \mathrm{~V}$. As $\mathrm{dAT}^{-}$can exchange five hydrogens, totally deuterated $\mathrm{dAT}^{-}$in labile positions presents an isotopic distribution with a major peak at $\mathrm{M}_{0}+5 \mathrm{~m} / \mathrm{z}$ [the zero subscript refers to a full $\mathrm{H}$ and full $\mathrm{C}_{12}$ molecule, and the added number indicates the number of extra nucleons ( $D$ or $\left.C_{13}\right)$ ], which is noted $\mathrm{M}_{0}+5$ (see point B in Figure 2). This isotopic peak was selected in the quadrupole before activation by collision with argon in the hexapole. To perform this activation, the acceleration voltage at the entrance of the hexapole was increased from $6 \mathrm{~V}$ (MS) to $25 \mathrm{~V}$ (MS/MS). All mass spectra were acquired and processed with MassLynx 4.0 (Service Pack 1, Micromass UK Ltd., Manchester, UK). All the peaks were centroided with suitable parameters to obtain a vertical line passing through the center of gravity of each isotopic peak. The resulting centered spectrum gave the centers of the isotopic peaks and their "areas." These latter correspond to the sum of the intensities of the points across the peak in the continuum trace.

\section{Infrared Multiphoton Dissociation Experiments}

These experiments were performed with a 9.4 tesla Apex-Qe FTICR mass spectrometer (Bruker Daltonics, Billerica, MA, USA) in negative ion mode. The dinucleotide solutions were infused via an external Apollo electrospray source at a flow rate of $180 \mu \mathrm{L} / \mathrm{h}$ with the assistance of $\mathrm{N}_{2}$ nebulizing gas. The off axis sprayer was grounded, the end-plate was set to $3 \mathrm{kV}$ and the inlet capillary was set to $3.5 \mathrm{kV}$. A $\mathrm{N}_{2}$ heated drying gas $\left(250{ }^{\circ} \mathrm{C}\right)$ flow assisted desolvation of ESI droplets. Ions were accumulated in the first hexapole for $1 \mathrm{~s}$, transferred through the mass-selective quadrupole (15 Da isolation window), and mass selectively accumulated in the second hexapole for $0.01 \mathrm{~s}$. The ions were transferred through high-voltage ion optics and captured by static trapping in the ICR cell. Before the IRMPD irradiation, the $\mathrm{M}_{0}+5$ isotopic peak of $\mathrm{dAT}^{-}$full $\mathrm{D}$ was isolated in the ICR cell by cleanup resonant ejections (cleanup shots) of undesired peaks. IRMPD was performed using a $25 \mathrm{~W}$ laser (Synrad, Mukilteo, WA, USA) with a wavelength of $10.6 \mu \mathrm{m}$. The laser beam passes through the center of the hollow dispenser cathode. Ions were irradiated for $100 \mathrm{~ms}$ at $15 \%$ and $20 \%$ laser power. All spectra were acquired with XMASS (version 7.0.8, Bruker Daltonics) in broadband mode with $512 \mathrm{k}$ data points and summed over 80 

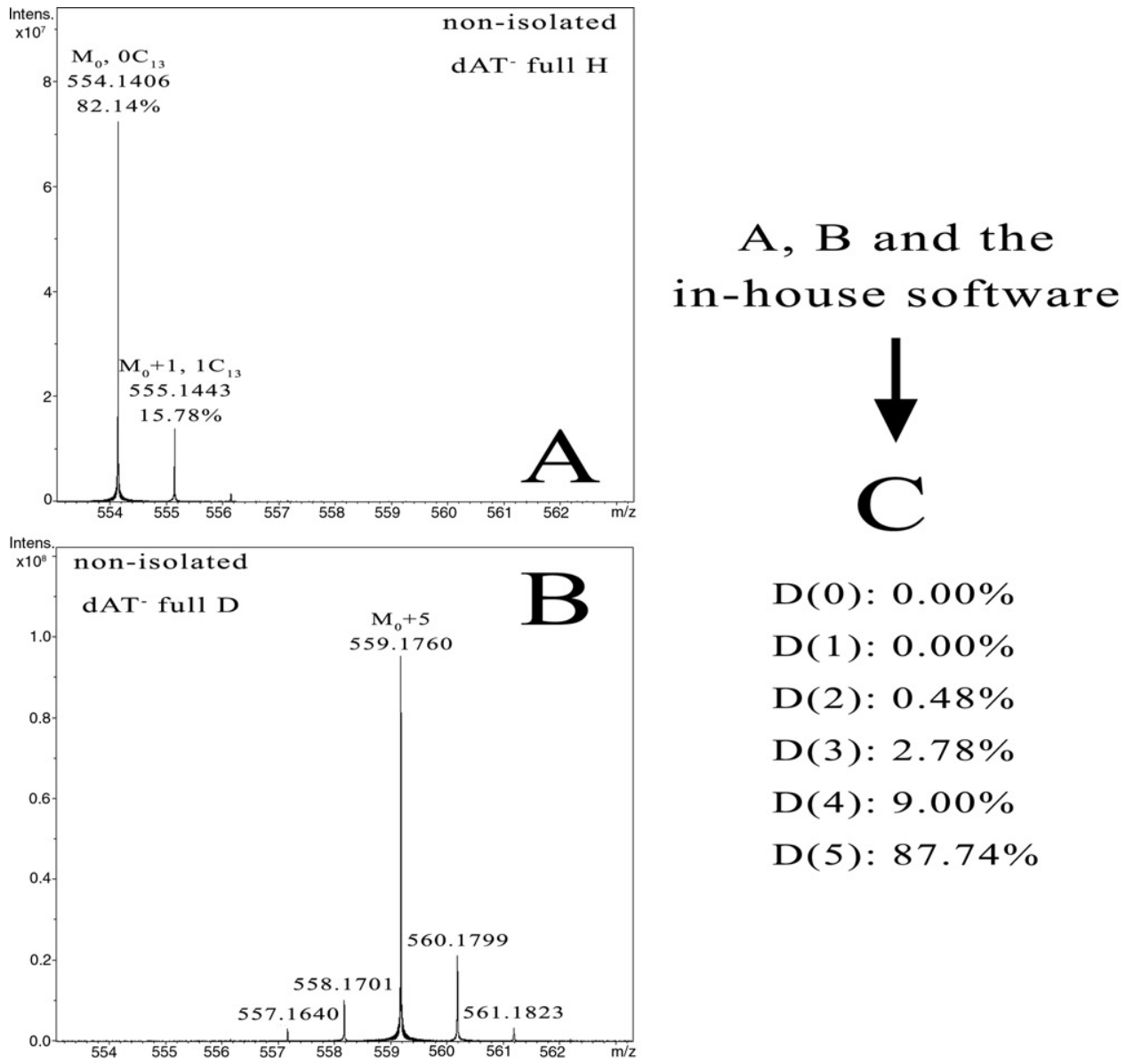

D(O): $0.00 \%$

$\mathrm{D}(1): 0.00 \%$

$\mathrm{D}(2): 0.48 \%$

$\mathrm{D}(3): 2.78 \%$

$\mathrm{D}(4): 9.00 \%$

$\mathrm{D}(5): 87.74 \%$

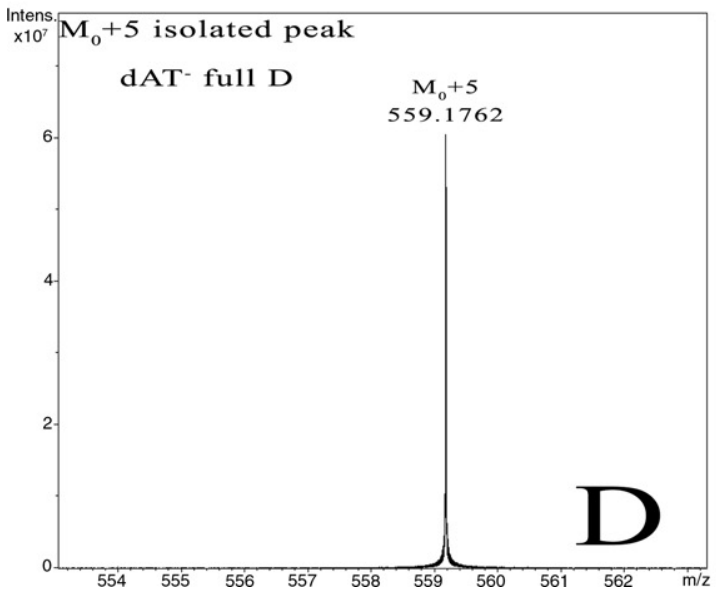

$A$ and $C$

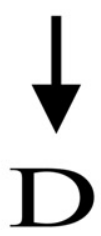

$\mathrm{D}(5) \times \mathrm{OC}_{13}=98.00 \%$

$\mathrm{D}(4) \times 1 \mathrm{C}_{13}=1.93 \%$

$\mathrm{D}(3) \times 2 \mathrm{C}_{13}=0.07 \%$

Figure 2. Illustration of the first step of the data processing.

scans. A mass list, in which $m / z$ values and peak heights are recorded, was created using DataAnalysis (version 3.4, Bruker Daltonics).

\section{Electron Induced Dissociation Experiments}

These experiments were performed with a 9.4 tesla Apex-Qe FTICR mass spectrometer (Bruker Daltonics) in negative ion mode as previously described for IRMPD with the exception that ions were accumulated in the first hexapole for $2.5 \mathrm{~s}$. The $\mathrm{M}_{0}+5$ isotopic peak was also isolated in the ICR cell by cleanup shots before electron irradiation. The electrons were emitted by a cylindrical indirectly heated hollow dispenser cathode. A heating current of 1.9 A was applied to the heater element located behind the cathode. A lens of $6 \mathrm{~mm}$ diameter located in front of the cathode ensures the focalization of the electron beam. The lens voltage was equal to $-18.8 \mathrm{~V}$ and the electrons were accelerated using a bias voltage of $-18.4 \mathrm{~V}$. The ions trapped in the ICR cell were subjected to $1 \mathrm{~s}$ irradiation by the electron beam. The spectra were summed over 500 scans. 


\section{Data Processing}

Step 1: Determination of the deuterated species that constitute the isotopic peak at $M_{0}+5$. The procedure is illustrated in Figure 2. First, the composition of deuterated species $\mathrm{D}(\mathrm{X})$ (X exchanged hydrogen atoms) was determined for the isotopic distribution of the non-isolated $\mathrm{dAT}^{-}$full D (point B in Figure 2). To do that, an in-house software was used. This software utilizes the known isotopic distribution (percentage of each peak obtained via the peak "areas" in CID and the peak heights in IRMPD and EID) of the non-deuterated ion (point A in Figure 2). Moreover, it is considered that the ion with a fixed number $X$, of exchanged hydrogens has the same isotopic distribution but shifted by $X / Z$ unit. The software determines the composition of each deuterated species (point $C$ in Figure 2) that best fits the experimental isotopic distribution (point B in Figure 2). Then, as the isotopic peak at $\mathrm{M}_{0}+5 \mathrm{~m} / \mathrm{z}$ corresponds to $\mathrm{D}(5)$ with zero $C_{13}$ atom, $D(4)$ with one $C_{13}$ atom, ... the isotopic distribution (percentage of each peak) of the non-deuterated dinucleotide ion and the composition of each deuterated species determined for the full deuterated ion were used to calculate the ratio of the deuterated species that constituted the isotopic peak at $\mathrm{M}_{0}+$ 5 (point D in Figure 2).

Step 2: Experimental and theoretical patterns of deuteration. As mentioned in the Introduction, the proposed mechanisms of fragmentation can be classified in two categories. Two models of fragmentation were considered according to these two categories. In the first model, the nonlabile protons that are bound to the carbon atoms of the ribose ring are proposed to be involved in the fragmentation. The second model involves the labile protons of the hydroxyl groups in $5^{\prime}$ (nucleoside A) and 3' (nucleoside T) on ribose moieties. According to both these models and knowing the ratio of the deuterated species that constitute the isotopic peak at $\mathrm{M}_{0}+5$, the theoretical deuteration patterns of the fragments were determined. Only the $D(5)$ with zero $C_{13}$ atom and the $D(4)$ with one $C_{13}$ atom species were considered, the $\mathrm{D}(3)$ with two $\mathrm{C}_{13}$ atoms species being always lower than $0.1 \%$ and so negligible. As $\mathrm{dAT}^{-}$has five exchangeable hydrogens, five different structures correspond to the $\mathrm{D}(4)$ with one $\mathrm{C}_{13}$ atom species (five positions for the non-exchanged hydrogen). Each of these structures can be divided into two cases according to the position of the $\mathrm{C}_{13}$ atom in the nucleoside $\mathrm{A}$ or in the nucleoside $\mathrm{T}$ (same number of carbon atoms in each nucleoside). In total, 10 structures correspond to the $\mathrm{D}(4)$ with one $\mathrm{C}_{13}$ atom species, each one existing with a probability of $1 / 10$ of the previously calculated ratio. The procedure followed to determine the theoretical deuteration patterns is illustrated in Figure 3 for the $D(5)$ with zero $C_{13}$ atom and two complementary $\mathrm{D}(4)$ with one $\mathrm{C}_{13}$ atom species. It should be noticed that for the neutral base loss fragments, the fact that the $\mathrm{C}_{13}$ atom can be in the base or in the ribose ring (same number of carbon atoms) is taken into account. These theoretical patterns were compared to those obtained using CID, IRMPD, and EID.

\section{Double Resonance Experiments}

Double resonance experiments consisted in continuous ejection of a specific fragment ion during the whole IRMPD or EID irradiation time. A modified experimental pulse program was used to perform these experiments. The pulse sequence for DR was the following: (1) start of ion ejection, (2) start of the IR or electron irradiation $10 \mu \mathrm{s}$ after (1), and (3) simultaneous stop of the irradiation and the ion ejection. The $\mathrm{m} / \mathrm{z}$ ratio of the ion to be ejected from the ICR cell was converted in its cyclotron frequency by the software, and the excitation voltage $\left(200 \mathrm{~V}_{p-p}\right)$ was attenuated by $10 \mathrm{~dB}$ in IRMPD and $20 \mathrm{~dB}$ in EID. For these experiments, the parent ion was the non-deuterated dinucleotide and the $\mathrm{M}_{0}$ isotopic peak at $\mathbf{M}_{0} \mathrm{~m} / \mathrm{z}$ was selected in the ICR cell thanks to cleanup shots before the IRMPD or EID irradiation.

\section{Results and Discussion}

\section{Fragmentation of the Totally Deuterated Dinucleotide $d A T^{-}$in Labile Positions in CID, IRMPD, and EID}

As suspected in the Introduction, the same fragments are observed in CID, IRMPD, and EID. These fragments are the neutral base $T$ loss $[M-T]$, the neutral base $A$ loss $[\mathrm{M}-\mathrm{A}]$, the $\mathrm{w}$ ion, the $\mathrm{d}$ ion, the neutral base $\mathrm{T}$ and A losses $[M-T-A]$, the $\left[\mathrm{w}-\mathrm{T}_{\text {neutral }}\right]$ and/or the $[\mathrm{d}-$ $\left.\mathrm{A}_{\text {neutral }}\right]$ ions (same mass), and the base $\mathrm{A}$ ion. They are observed with different ratios in the different fragmentation methods. Despite the fact that the fragments are the same, their deuteration patterns change according to the used fragmentation technique. This is reported fragment by fragment in the following paragraphs.

Supplemental Data: The deuteration patterns of the neutral base T loss fragment; the deuteration patterns of the $\mathrm{d}$ ion; the full CID, IRMPD, and EID mass spectra, can be found in the electronic version of this article.

\section{The Neutral Base A Loss}

As illustrated in Figure 4a, the experimental CID deuteration pattern is similar to the theoretical one obtained considering that the nonlabile protons are involved in the fragmentation process. The CID pattern shows a major peak at $[\mathrm{M}-\mathrm{A}]_{0}+3 \mathrm{~m} / \mathrm{z}$, which corresponds to $\mathrm{A}-\mathrm{H}$ loss from the fully deuterated dinucleotide ion in labile positions (the zero subscript refers to a full $\mathrm{H}$ and full $\mathrm{C}_{12}$ molecule, and the added number indicates the number of extra nucleons $\left(\mathrm{D}\right.$ or $\left.\mathrm{C}_{13}\right)$ ). Quite the reverse, the experimental IRMPD and EID deuteration patterns 


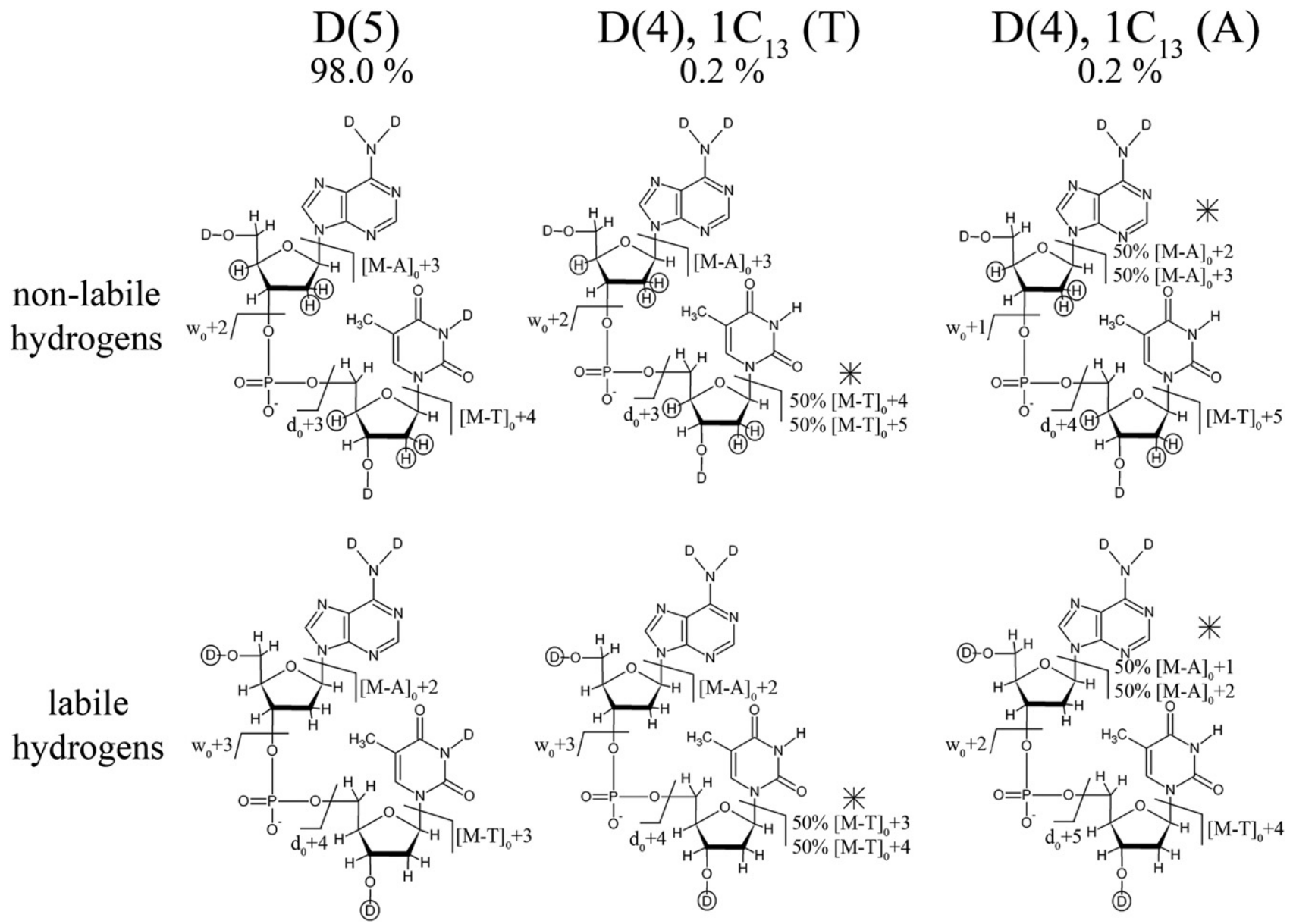

Figure 3. Illustration of the second step of the data processing: the hydrogen or deuterium atoms involved in the mechanisms are circled and for each fragment, the added number indicates the number of extra nucleons $\left(\mathrm{D}\right.$ or $\left.\mathrm{C}_{13}\right)$ in comparison to the obtained fragment for the non-deuterated and full $\mathrm{C}_{12}$ dinucleotide. The asterisk is placed at the level of the nucleoside that possesses the $C_{13}$ atom.

show a major peak at $[\mathrm{M}-\mathrm{A}]_{0}+2 \mathrm{~m} / \mathrm{z}$ should have in agreement with the involvement of labile protons in the fragmentation process. The A base takes a deuterium to be lost as a neutral group. It is important to notice that the loss of A-D can also be explained with a process involving nonlabile protons if an intramolecular migration (scrambling) of labile and nonlabile protons takes place. The ribose moiety fixed to the A base possesses eight hydrogens including one labile, exchangeable. If there is scrambling, the deuterium has a probability of $1 / 8$ to be at each position. Considering the 1,2-elimination mechanism where one of the two protons at the $2^{\prime}$ position on the ribose is lost with the base, the peak at $[\mathrm{M}-\mathrm{A}]_{0}+2 \mathrm{~m} / \mathrm{z}$ should have should have at the most an intensity of $25 \%$ which is much lower than observed. It is assumed that no scrambling of labile and nonlabile protons takes place. The experimental deuteration patterns cannot be explained with only one mechanism. The two proposed categories of mechanisms can be considered as different fragmentation channels and must coexist to form the neutral base A loss fragment. Moreover as the deuteration pattern varies from one fragmentation technique to another, the different frag- mentation channels coexist but are taken with different ratios for each MS/MS method.

\section{The Neutral Base T Loss}

Unlike the loss of neutral A base, the experimental deuteration patterns are the same for all the fragmentation methods. They correspond to the theoretical pattern that involves nonlabile protons in the fragmentation process. The fragmentation channel where the $\mathrm{T}$ base takes a proton to be lost as a neutral group is the only accessible channel and is, therefore, the one used in all the chosen MS/MS techniques.

\section{The w Ion}

For all the fragmentation methods (Figure $4 b$ ), the experimental deuteration pattern is more similar to the theoretical one obtained considering that the nonlabile protons are involved in the fragmentation process. As mentioned in the Introduction, the neutral base loss is proposed to be the first step of the fragmentation process for CID and IRMPD. In this case and if the A 
CID
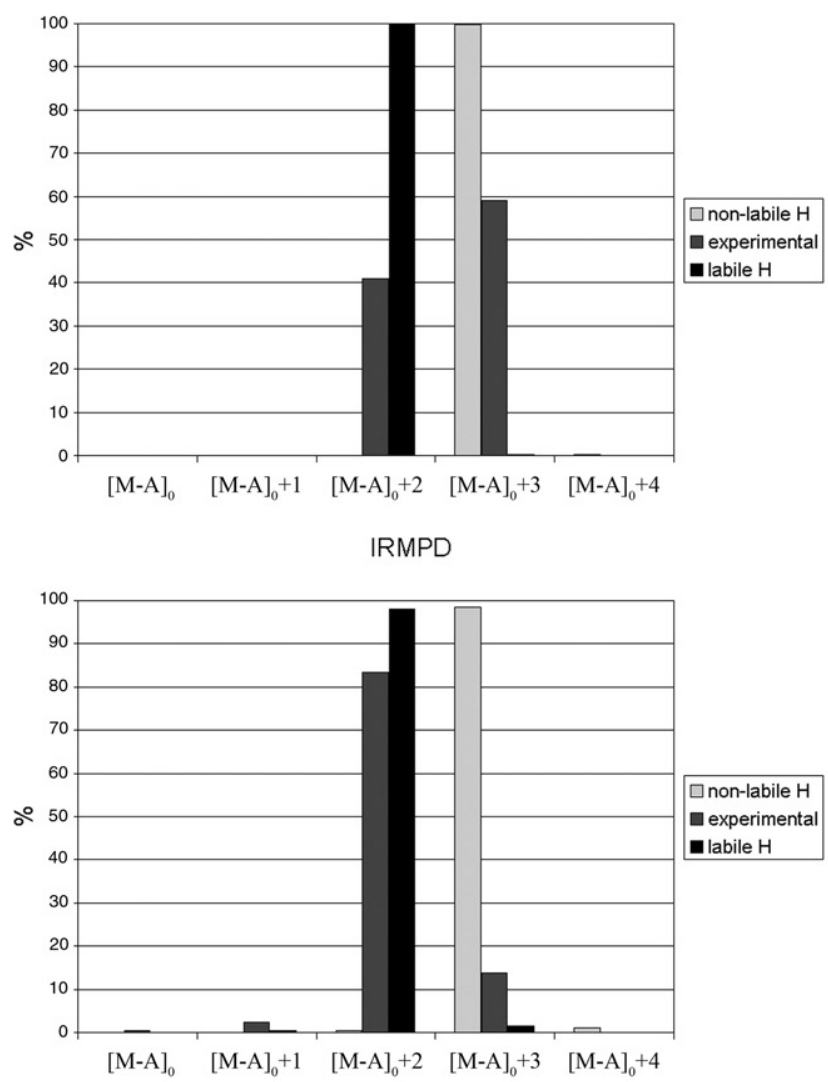

EID

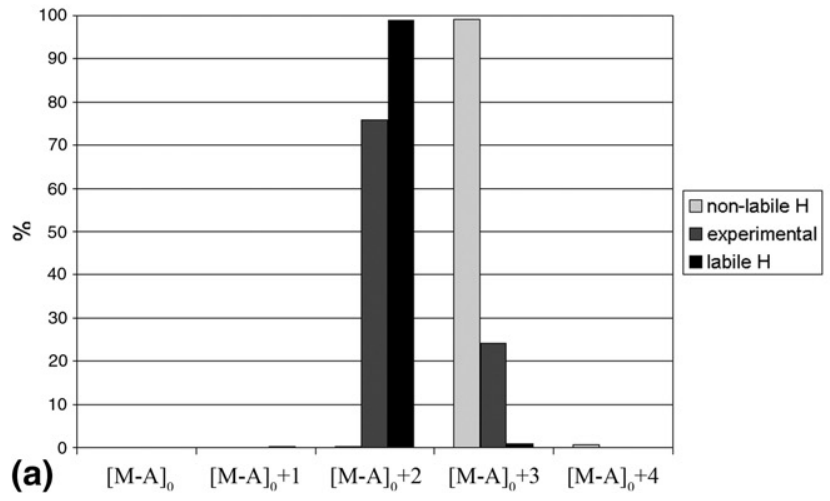

CID
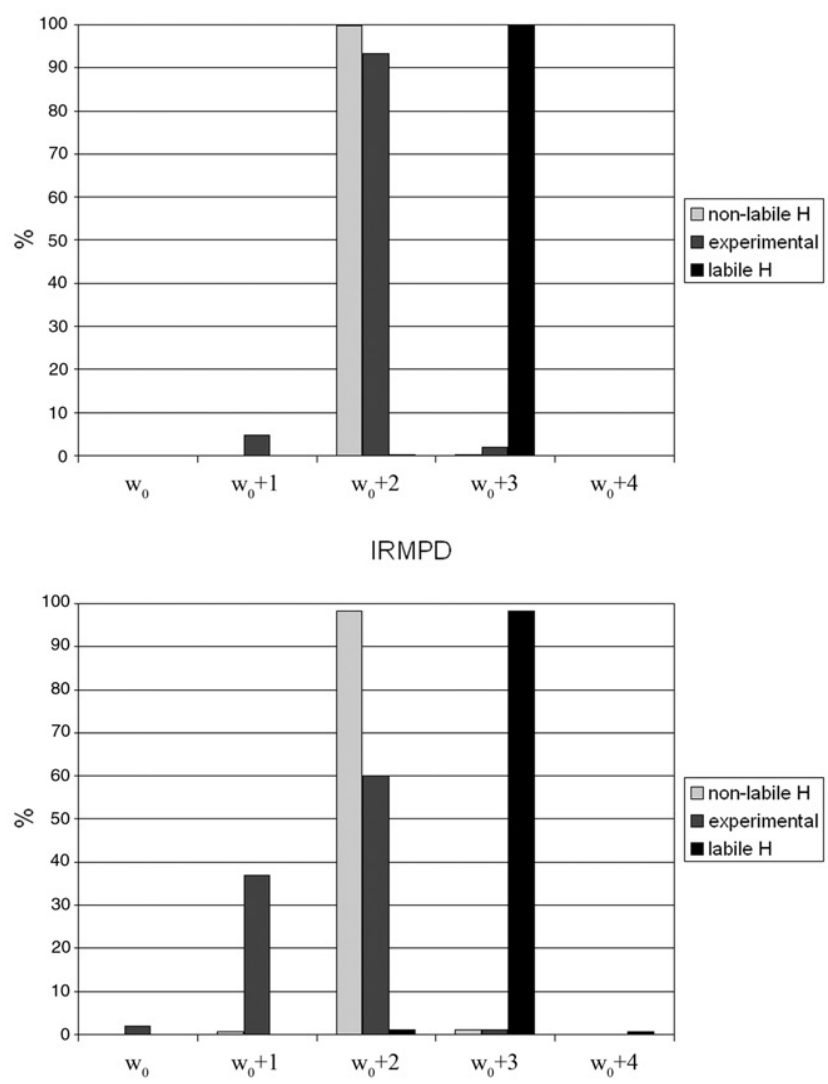

EID

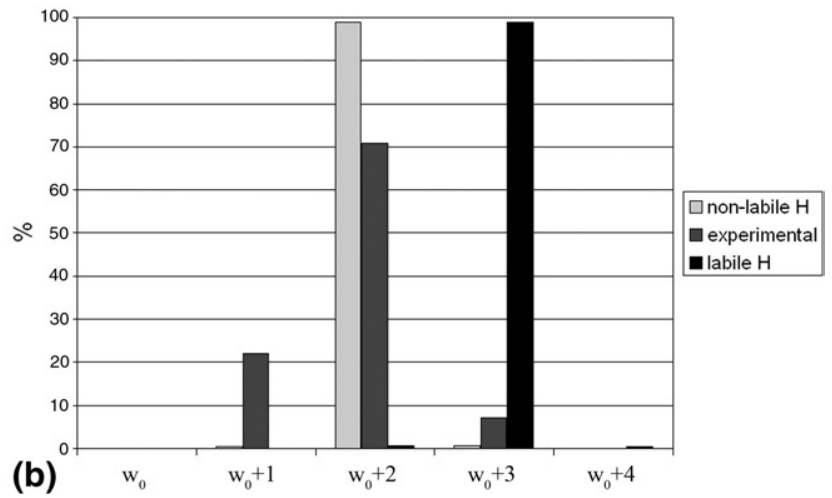

Figure 4. The deuteration patterns of the neutral base A loss fragment (a) and the $\mathrm{w}$ ion (b).

base takes the deuterium of the 5'-hydroxyl group to be lost as a neutral species, the $\mathrm{w}$ ion has no other choice than to take a proton of the ribose ring (with subsequent double-bond generation) to be formed. As the A base significantly leaves with a deuterium, this could explain the low intensity of the peak at $\mathrm{w}_{0}+3 \mathrm{~m} / \mathrm{z}$. To check the possibility of neutral A loss as the first step of the fragmentation, double resonance experiments have been performed in IRMPD and EID. Their results are presented and discussed later in this section.

Another important result is the observation of a significant amount of ions at $\mathrm{w}_{0}+1 \mathrm{~m} / \mathrm{z}$ mostly in IRMPD and EID (Figure $4 b$ ). This $\mathrm{w}_{0}+1$ ion corre- sponds to a $\mathrm{w}$ fragment with only one deuterium. It is one less deuterium compared with the theoretical $\mathrm{w}$ ion obtained considering that nonlabile protons are involved in the fragmentation process. One scenario can explain this observation: if the $\mathrm{w}$ ion is formed from the neutral base A loss, if the A base takes the deuterium at the $3^{\prime}$-hydroxyl position on the ribose of the $\mathrm{T}$ nucleoside, and if the $\mathrm{w}$ ion takes a proton to be formed, the $\mathrm{w}$ ion will present a peak at $\mathrm{w}_{0}+1 \mathrm{~m} / \mathrm{z}$. The second condition is checked thanks to the analysis of the different conformations adopted by the dinucleotide. These conformations were investigated by dynamic simulations that were carried out with 
Yasara (YASARA Biosciences and CMBI Outstation Austria, http://www.yasara.org). Amber99 was used as the force field. The distances (Figure 5) between the hydrogen atom of the $3^{\prime}$-hydroxyl group (ribose of the $\mathrm{T}$ nucleoside) and the different nitrogen atoms of the $\mathrm{A}$ base rings were monitored during the dynamic simulations (100 ns at $295 \mathrm{~K})$. The distances d1, and especially $\mathrm{d} 3$, were often between 1.8 and $2 \AA$, which is characteristic for a hydrogen bond. A hydrogen transfer from the $3^{\prime}$-hydroxyl group to the A base is probable via either $\mathrm{d} 3$ or $\mathrm{d} 1$. The nitrogen of the $\mathrm{N}$-glycosidic bond does not seem to be included in this transfer as the distance noted, d4, was always higher than $3 \AA$, which is not small enough for a direct interaction. From these dynamic simulations, it can be concluded that the A base is allowed to take the deuterium of the $3^{\prime}$-hydroxyl group to be lost as a neutral fragment. These calculations also support the idea that the fragmentation mechanisms involving labile protons require peculiar three-dimensional structures that locate the labile pro- ton donor and acceptor sites close to each other. As just previously mentioned, the first condition is checked thanks to DR experiments that are discussed later in this section.

\section{The d Ion}

In IRMPD, the $d$ ion was not intense enough to be exploited. Experimental deuteration patterns were only obtained in CID and EID. These patterns are more similar to the theoretical one obtained considering that the labile protons are involved in the fragmentation process. The $\mathrm{d}$ ion mostly takes the deuterium of the $3^{\prime}$-hydroxyl group to be formed. The observation of a peak at $\mathrm{d}_{0}+3 \mathrm{~m} / \mathrm{z}$ can be justified by the fact that the fragmentation channel involving nonlabile protons also exists.

The fragment that corresponds to the losses of both the neutral bases $\mathrm{A}$ and $\mathrm{T}$ presents deuteration patterns that agree with the sum of the neutral base $\mathrm{A}$

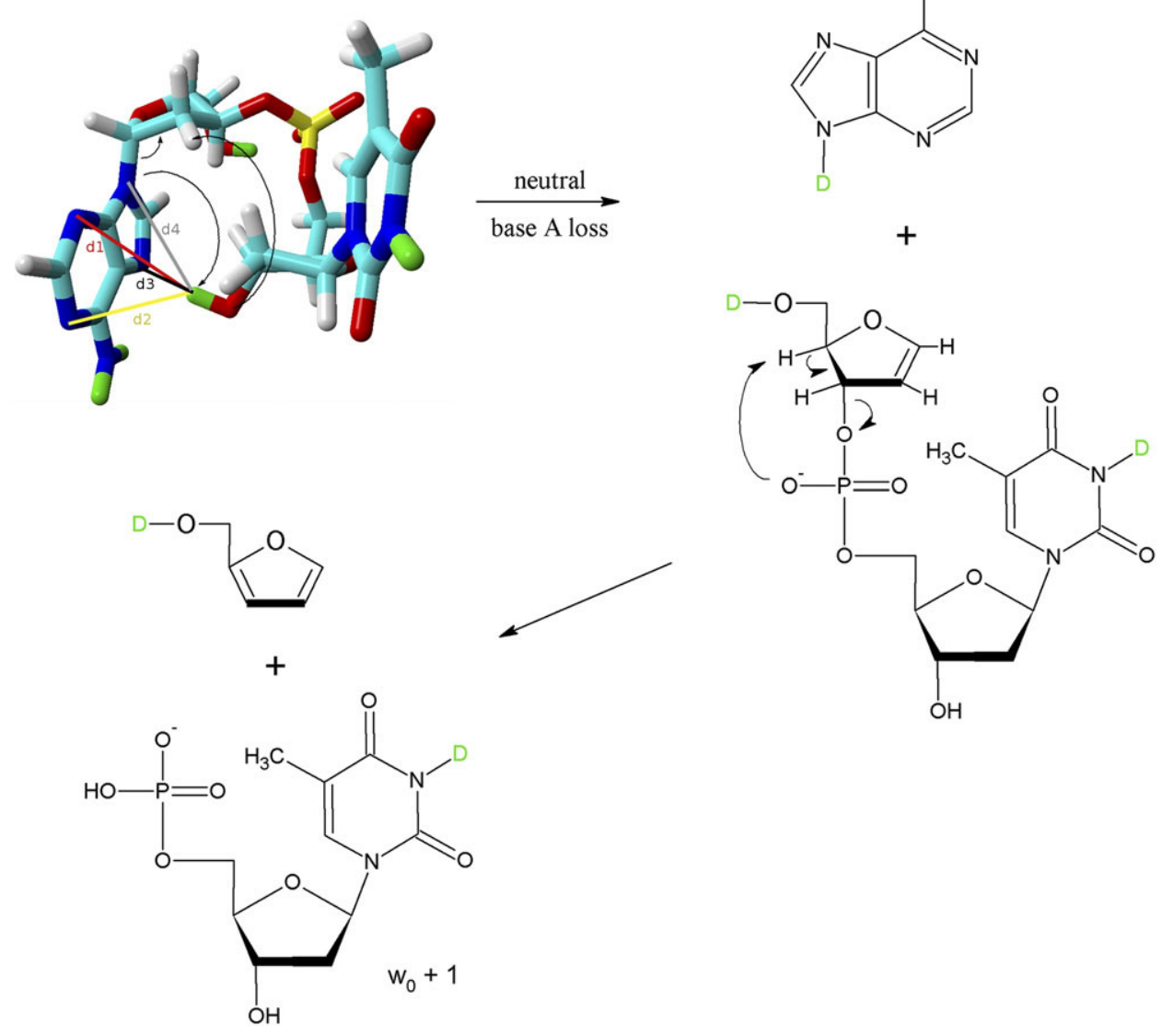

Figure 5. A relevant structure of $\mathrm{dAT}^{-}$obtained during dynamic simulations followed by the mechanism (in two dimensions) leading to the $\mathrm{w}_{0}+1$ ion formation. The distances $(\mathrm{d} 1, \mathrm{~d} 2, \mathrm{~d} 3$, and $\mathrm{d} 4$ ) between the hydrogen atom of the $3^{\prime}$-hydroxyl group on the ribose of the $\mathrm{T}$ nucleoside and the different nitrogen atoms of the A base rings are shown, and the deuterium atoms are highlighted in green. Even if an arrow goes from the nitrogen of the N-glycosidic bond to the 3'-hydroxyl group, this nitrogen atom does not seem to be involved in the initial transfer of the proton (see the Results and Discussion section). 
loss and the neutral base $\mathrm{T}$ loss patterns. This was expected as the $[\mathrm{M}-\mathrm{A}-\mathrm{T}]$ fragment most probably comes from the neutral base $\mathrm{T}$ loss from the $[\mathrm{M}-\mathrm{A}]$ fragment and the neutral base A loss from the $[\mathrm{M}-\mathrm{T}]$ fragment with the same fragmentation channels followed as previously proposed.

The fragment ion corresponding to the $\left[\mathrm{w}-\mathrm{T}_{\text {neutral }}\right]$ and/or the $\left[\mathrm{d}-\mathrm{A}_{\text {neutral }}\right]$ ions was not analyzed, these theoretical deuteration patterns being too complicated to generate (too many possibilities). As expected, the $\mathrm{A}^{-}$ion was only observed with two deuteriums.

All the fragmentation experiments of the totally deuterated dinucleotide in labile positions show clearly the coexistence of several fragmentation channels. These can be classified according to the involvement of nonlabile (on ribose ring) or labile (hydroxyl groups) protons in the fragmentation process. For the proton transfer to take place, a spatial closeness between donor and acceptor sites is needed. For the fragmentation mechanisms that imply the transfer of nonlabile protons, this closeness preexists. But for those involving the transfer of labile protons, a conformational rearrangement of the molecule has to happen to place the donor site near that of the acceptor. Such particular threedimensional structures are observed for a dynamic simulation of $100 \mathrm{~ns}: 0.3 \%$ of the time for the A base and the 5'-hydroxyl group (ribose of the A nucleoside), and $9 \%$ of the time for the A base and the 3 '-hydroxyl group (ribose of the $\mathrm{T}$ nucleoside). Moreover, when considering the bonds involved in the proton transfers, the transfers involving nonlabile protons are suspected to need more energy than those involving labile protons. The transfer of labile protons could therefore be encountered when the internal energy is increased in small steps. The fragmentation channels that involve nonlabile protons are entropically favorable and enthalpically less favorable. Conversely, the fragmentation channels that involve labile protons are enthalpically favorable and entropically less favorable. As the deuteration pattern varies from a MS/MS technique to another, the different fragmentation channels coexist but are taken with different ratios according to the fragmentation method used. This can be discussed regarding the transferred energy under activation and the accessible experimental time scale of each MS/MS technique. For CID in a Q-TOF instrument, the transferred energy under activation is higher than in IRMPD and EID and the time scale is relatively short going from $10^{-5}$ to $10^{-3} \mathrm{~s}$. In IRMPD and EID, the ions are activated during respectively $10^{-1}$ and $1 \mathrm{~s}$, and the transferred energy under activation is relatively low. According to the RRKM theory, it can be deduced that in CID fragmentation, the channels which are entropically favorable and enthalpically less favorable are characterized by higher rate constants than those which are enthalpically favorable and entropically less favorable. The opposite is found in IRMPD and EID (lowenergy activation methods); the channels which are enthalpically favorable and entropically less favorable are characterized by higher rate constants. This agrees with the findings of the experiments as the fragments coming from the labile proton fragmentation channels are observed more in IRMPD and EID (see [M - A] ion, observation of $\mathbf{w}_{0}+1 \mathrm{~m} / \mathrm{z} \ldots$.).

\section{Double Resonance Experiments}

Double resonance experiments were performed in IRMPD and EID with the aim of checking the existence of fragmentation channels where the neutral base loss would be the first step. The selected $C_{12}$ isotope of the non-deuterated dinucleotide anion was activated in IRMPD or EID and the mono-isotopic $\left(\mathrm{M}-\mathrm{AH}-\mathrm{H}^{+}\right)^{-}$ fragment ion was continuously ejected during the irradiation.

\section{DR-IRMPD}

First, it was not possible to completely eject the [M - A] fragment despite the length and the intensity of the resonant shot. The $[\mathrm{M}-\mathrm{A}]$ fragment intensity (stated in percent regarding the parent ion intensity) was decreased 8.8 and 4.1 times, respectively, at $15 \%$ and $20 \%$ laser power, which was expected as the $[\mathrm{M}-\mathrm{A}]$ fragment is more produced at $20 \%$ laser power. The fragment intensities without and with double resonance at $15 \%$ and $20 \%$ laser power are shown in Figure $6 a$ and $b$.

The $[\mathrm{M}-\mathrm{T}]$ fragment intensity decreases during DR experiments even though its formation does not depend on the $[\mathrm{M}-\mathrm{A}]$ fragment one. The $[\mathrm{M}-\mathrm{T}]$ fragment $(428.1 \mathrm{~m} / \mathrm{z})$ having a mass near to the [M - A] fragment one $(419.1 \mathrm{~m} / \mathrm{z})$ and considering the low attenuation (10 $\mathrm{dB}$ ) of the resonant shot, the [M - T] fragment is probably a bit resonantly activated by side activation bands.

The $\mathrm{d}$ fragment is observed at $20 \%$ laser power, not at $15 \%$. Its intensity is decreased three times even though its formation does not depend on the [M $-\mathrm{A}]$ fragment one. This decrease can be explained not by side activation bands because the $\mathrm{d}$ fragment mass $(330.1 \mathrm{~m} / \mathrm{z})$ is quite different from the $[\mathrm{M}-\mathrm{A}]$ fragment one, but by the fact that the $d$ fragment could be formed from the $[\mathrm{M}-\mathrm{T}]$ fragment.

The $\mathrm{w}$ fragment intensity is decreased 14.6 and 8.2 times at, respectively, 15\% and 20\% laser power. These decreases are quite important, which prove that at least some $\mathrm{w}$ ions originate from the decomposition of the $\left(\mathrm{M}-\mathrm{AH}-\mathrm{H}^{+}\right)^{-}$ion. In double resonance experiments, the observation that a fragment is reduced but does not totally disappear when its suspected parent ion is ejected can be interpreted in different ways. In this case, an explanation is easily found as the [M - A] ion is not totally ejected but other justifications exist: (1) several ion formation channels coexist, and (2) the fragment is formed and detected before the ejection is complete. This second justification corresponds to a limitation of double resonance experiments. To be freed from this limitation, the time it takes to eject an ion by 

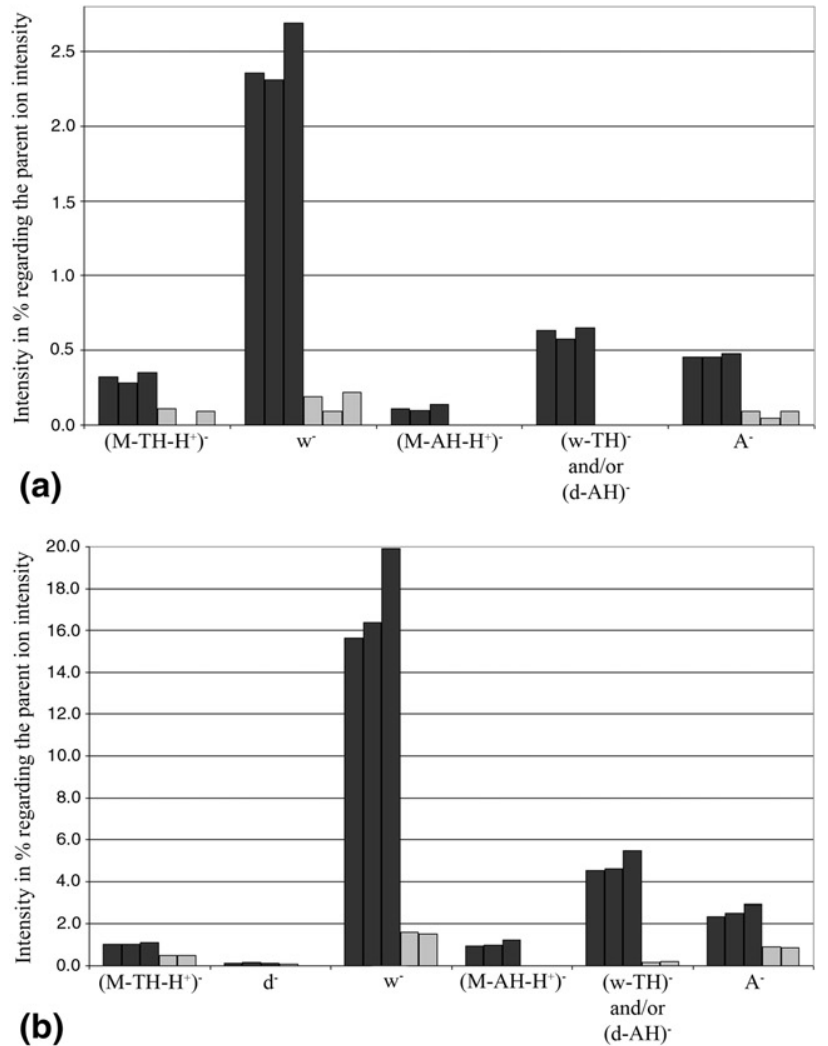

(b)

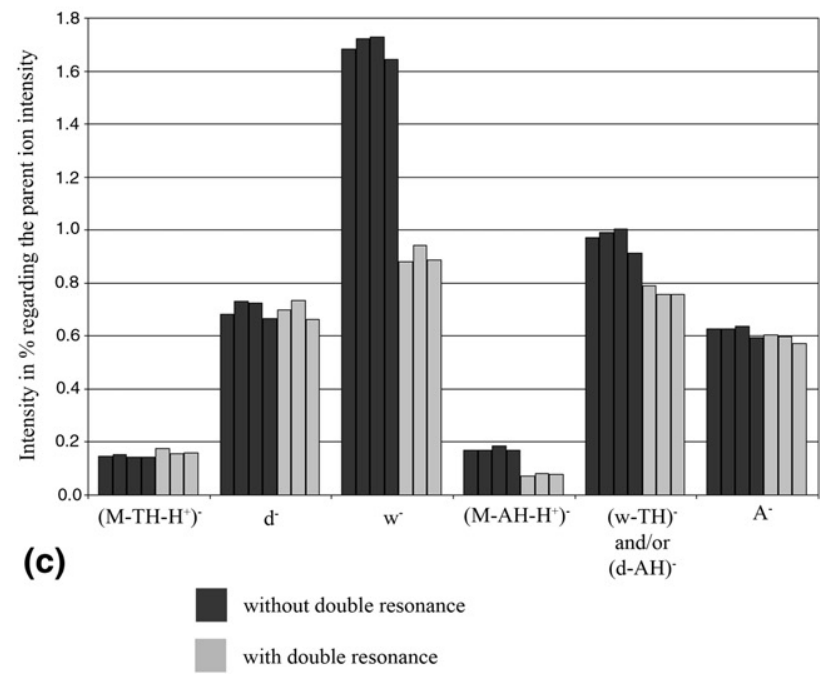

Figure 6. The fragment intensities without and with double resonance (a) at $15 \%$ laser power in IRMPD, (b) at $20 \%$ laser power in IRMPD, and (c) in EID.

resonant ejection must be much faster than the time required for the consecutive products to be formed from the ejected ion.

As expected, the $[\mathrm{M}-\mathrm{A}-\mathrm{T}]$ fragment decreases and even disappears. This disappearance can be explained by the fact that the $[\mathrm{M}-\mathrm{A}]$ and also the $[\mathrm{M}-$ $\mathrm{T}]$ ions are ejected by the resonant shot and by the low intensity of the $[\mathrm{M}-\mathrm{A}-\mathrm{T}]$ fragment.

The $(\mathrm{w}-\mathrm{TH})^{-}$and/or the $(\mathrm{d}-\mathrm{AH})^{-}($same mass) fragment totally disappears at $15 \%$ laser power and is decreased 19.9 times at $20 \%$ laser power. This is explained in the same way as for the $[\mathrm{M}-\mathrm{A}-\mathrm{T}]$ fragment. The $\mathrm{A}^{-}$fragment is decreased 6 and 2.2 times at respectively $15 \%$ and $20 \%$ laser power. Some $\mathrm{A}^{-}$ions are linked to the $\left(\mathrm{M}-\mathrm{AH}-\mathrm{H}^{+}\right)^{-}$ion formation.

\section{DR-EID}

Unlike DR-IRMPD experiments, the $\left(\mathrm{M}-\mathrm{AH}-\mathrm{H}^{+}\right)^{-}$ ion was completely ejected, which was expected as the $[\mathrm{M}-\mathrm{A}]$ fragment is less intense in EID that in IRMPD. As illustrated in Figure 6c, no influence of double resonance is observed for the $\left(\mathrm{M}-\mathrm{TH}-\mathrm{H}^{+}\right)^{-}$, the $\mathrm{d}$ and the $\mathrm{A}^{-}$ions.

The $\mathrm{w}$ ion intensity is decreased 2.5 times which means that at least some of the $\mathrm{w}$ ions originate from the decomposition of the $\left(\mathrm{M}-\mathrm{AH}-\mathrm{H}^{+}\right)^{-}$fragment. As previously mentioned the observation that the $\mathrm{w}$ fragment is reduced but does not totally disappear is interpreted in two ways: (1) several fragmentation channels coexist for the formation of the $\mathrm{w}$ ion, and/or (2) the $\mathrm{W}$ ion is formed and detected before the ejection of $\left(\mathrm{M}-\mathrm{AH}-\mathrm{H}^{+}\right)^{-}$is complete. This was expected because by being linked to the $[\mathrm{M}-\mathrm{A}]$ or $\mathrm{w}$ ion, the $\left(\mathrm{M}-\mathrm{AH}-\mathrm{TH}-\mathrm{H}^{+}\right)^{-}$and the $(\mathrm{w}-\mathrm{TH})^{-}$ion intensities are decreased 3 and 1.7 times in double resonance experiments.

All these double resonance experiments show that there are fragmentation channels that correspond to consecutive fragmentation processes. In particular, some $\mathrm{w}$ ions originate from the decomposition of the $\left(\mathrm{M}-\mathrm{AH}-\mathrm{H}^{+}\right)^{-}$fragment ion. The DR influence seems to be more important in IRMPD than in EID. This is similar to that shown by Kinet et al. [22] and Yang et al. [23]. However, the non-complete ejection of the (M $\left.\mathrm{AH}-\mathrm{H}^{+}\right)^{-}$ion in IRMPD and the fact that the ejection and ion formation times are unknown prevent this from being affirmed.

\section{Conclusions}

The fragmentation of a totally deuterated dinucleotide in labile positions demonstrates the coexistence of several fragmentation channels that can be classified in relation to the involvement of the nonlabile or labile protons in the fragmentation process. These channels are taken with different ratios according to the used MS/MS technique, which means according to the transferred energy under activation and the accessible time scale associated with this method. Fragmentation channels involving labile protons are enthalpically favorable and entropically less favorable as they require particular three-dimensional conformations of the dinucleotide. The involvement of labile protons in the fragmentation mechanisms are more observed in IRMPD and EID.

Double resonance experiments demonstrate the existence of consecutive fragmentation processes. The 
probability that such processes happen depends also on the MS/MS technique used.

The coexistence of so many fragmentation channels does not make obvious the localization by MS/MS of the incorporated deuteriums in oligonucleotides. Further to particular conformations that are adopted by the oligonucleotide, exchangeable protons can be involved in the fragmentation process. In this case, labile protons are no longer at their initial positions and the localization results obtained by tandem mass spectrometry are distorted.

\section{Acknowledgments}

The authors acknowledge support for this work by the FRS-FNRS (Fonds de la Recherche Scientifique-FNRS), Belgium.

\section{Appendix A Supplementary Material}

Supplementary material associated with this article may be found in the online version at doi:10.1016/ j.jasms.2009.08.004.

\section{References}

1. Jorgensen, T. J. D.; Gardsvoll, H.; Ploug, M.; Roepstorff, P. Intramolecular Migration of Amide Hydrogens in Protonated Peptides Upon Collisional Activation. J. Am. Chem. Soc. 2005, 127, 2785-2793.

2. Rand, K. D.; Jorgensen, T. J. D. Development of a Peptide Probe for the Occurrence of Hydrogen $\left({ }^{1} \mathrm{H} /{ }^{2} \mathrm{H}\right)$ Scrambling Upon Gas-Phase Fragmentation. Anal. Chem. 2007, 79, 8686-8693.

3. Rand, K. D.; Adams, C. M.; Zubarev, R. A.; Jorgensen, T. J. D. Electron Capture Dissociation Proceeds with a Low Degree of Intramolecular Migration of Peptide Amide Hydrogens. J. Am. Chem. Soc. 2008, 130, 1341-1349.

4. Bache, N.; Rand, K. D.; Roepstorff, P.; Jorgensen, T. J. D. Gas-Phase Fragmentation of Peptides by MALDI In-Source Decay with Limited Amide Hydrogen $\left({ }^{1} \mathrm{H} /{ }^{2} \mathrm{H}\right)$ Scrambling. Anal. Chem. 2008, 80, 6431-6435.

5. Zehl, M.; Rand, K. D.; Jensen, O. N.; Jorgensen, T. J. D. Electron Transfer Dissociation Facilitates the Measurement of Deuterium Incorporation into Selectively Labeled Peptides with Single Residue Resolution. J. Am. Chem. Soc. 2008, 130, 17453-17459.

6. Wu, J.; McLuckey, S. A. Gas-Phase Fragmentation of Oligonucleotide Ions. Int. J. Mass Spectrom. 2004, 237, 197-241.

7. Cerny, R. L.; Gross, M. L.; Grotjahn, L. Fast Atom Bombardment Combined with Tandem Mass Spectrometry for the Study of Dinucleotides. Anal. Biochem. 1986, 156, 424-435.

8. Phillips, D. R.; McCloskey, J. A. A Comprehensive Study of the Low Energy Collision-Induced Dissociation of Dinucleoside Monophosphates. Int. J. Mass Spectrom. Ion Proceesses 1993, 128, 61-82.
9. McLuckey, S. A.; Habibi-Goudarzi, S. Decompositions of MultiplyCharged Oligonucleotide Anions. J. Am. Chem. Soc. 1993, 115, 1208512095 .

10. Rodgers, M. T.; Campbell, S.; Marzluff, E. M.; Beauchamp, J. L. LowEnergy Collision-Induced Dissociation of Deprotonated DinucleotidesDetermination of the Energetically Favored Dissociation Pathways and the Relative Acidities of the Nucleic-Acid Bases. Int. J. Mass Spectrom. Ion Processes 1994, 137, 121-149.

11. Barry, J. P.; Vouros, P.; Vanschepdael, A.; Law, S. J. Mass and Sequence Verification of Modified Oligonucleotides Using Electrospray Tandem Mass-Spectrometry. J. Mass Spectrom. 1995, 30, 993-1006.

12. Nordhoff, E.; Karas, M.; Cramer, R.; Hahner, S.; Hillenkamp, F.; Kirpekar F.; Lezius, A.; Muth, J.; Meier, C.; Engels, J. W. Direct Mass-Spectrometric Sequencing of Low-Picomole Amounts of Oligodeoxynucleotides with up to 21 Bases by Matrix-Assisted Laser-Desorption Ionization MassSpectrometry. J. Mass Spectrom. 1995, 30, 99-112.

13. Zhu, L.; Parr, G. R.; Fitzgerald, M. C. Nelson, C. M.; Smith, L. M Oligodeoxynucleotide Fragmentation in MALDI/TOF Mass-Spectrometry Using 355-Nm Radiation. J. Am. Chem. Soc. 1995, 117, 6048-6056.

14. Wang, Z.; Wan, K. X.; Ramanathan, R.; Taylor, J. S.; Gross, M. L. Structure and Fragmentation Mechanisms of Isomeric T-Rich Oligodeoxynucleotides: A Comparison of Four Tandem Mass Spectrometric Methods. J. Am. Soc. Mass Spectrom. 1998, 9, 683-691.

15. Gross, J.; Hillenkamp, F.; Wan, K. X.; Gross, M. L. Metastable Decay of Negatively Charged Oligodeoxynucleotides Analyzed with Ultraviolet Matrix-Assisted Laser Desorption/Ionization Post-Source Decay and Deuterium Exchange. J. Am. Soc. Mass Spectrom. 2001, 12, 180-192.

16. Wan, K. X.; Gross, J.; Hillenkamp, F.; Gross, M. L. Fragmentation Mechanisms of Oligodeoxynucleotides Studied by H/D Exchange and Electrospray Ionization Tandem Mass Spectrometry. J. Am. Soc. Mass Spectrom. 2001, 12, 193-205.

17. Wan, K. X.; Gross, M. L. Fragmentation Mechanisms of Oligodeoxynucleotides: Effects of Replacing Phosphates with Methylphosphonates and Thymines with Other Bases in T-Rich Sequences. J. Am. Soc Mass Spectrom. 2001, 12, 580-589.

18. Liu, J.; Cao, S.; Jia, B.; Wei, D.; Liao, X.; Lu, J.; Zhao, Y. A Theoretical and Mass Spectrometry Study of the Novel Mechanism of N-Glycosidic Bond Cleavage in Nucleoside. Int. J. Mass Spectrom. 2009, 282, 1-5.

19. Balbeur, D.; Dehareng, D.; De Pauw, E. Conformationally Driven Gas-Phase H/D Exchange of Dinucleotide Negative Ions. J. Am. Soc. Mass Spectrom. 2007, 18, 1827-1834.

20. Sannes-Lowery, K. A. Hofstadler, S A Sequence Confirmation of Modified Oligonucleotides Using IRMPD in the External Ion Reservoir of an Electrospray Ionization Fourier Transform Ion Cyclotron Mass Spectrometer. J. Am. Soc. Mass Spectrom. 2003, 14, 825-833.

21. Keller, K. M.; Brodbelt, J. S. Collisionally Activated Dissociation and Infrared Multiphoton Dissociation of Oligonucleotides in a Quadrupole Ion Trap. Anal. Biochem. 2004, 326, 200-210.

22. Kinet, C.; Gabelica, V.; Balbeur, D.; De Pauw, E. Electron Detachmen Dissociation (EDD) Pathways in Oligonucleotides. Int. J. Mass Spectrom. in press.

23. Yang, J.; Hakansson, K. Characterization of Oligodeoxynucleotide Fragmentation Pathways in Infrared Multiphoton Dissociation and Electron Detachment Dissociation by Fourier Transform Ion Cyclotron Double Resonance. Eur. J. Mass Spectrom. 2009, 15, 293-304.

24. Wang, B. H.; McLafferty, F. W. Electron-Impact Excitation of Ions from Larger Organic-Molecules. Org. Mass Spectrom. 1990, 25, 554-556.

25. Lioe, H.; O'Hair, R. A. J. Comparison of Collision-Induced Dissociation and Electron-Induced Dissociation of Singly Protonated Aromatic Amino Acids, Cystine and Related Simple Peptides Using a Hybrid Linear Ion Trap-FT-ICR Mass Spectrometer. Anal. Bioanal. Chem. 2007, 389, 1429-1437.

26. Wolff, J. J.; Laremore, T. N.; Aslam, H.; Linhardt, R. J.; Amster, I. J. Electron-Induced Dissociation of Glycosaminoglycan Tetrasaccharides. J. Am. Soc. Mass Spectrom. 2008, 19, 1449-1458. 